\title{
Lowering the energy threshold of large-mass bolometric detectors
}

\author{
S. Di Domizio ${ }^{a}$, F. Orio ${ }^{b}$ and M. Vignati ${ }^{b *}$ \\ ${ }^{a}$ Università di Genova and Sezione INFN di Genova, Genova I-16146, Italy \\ ${ }^{b}$ Sapienza Università di Roma and Sezione INFN di Roma, Roma I-00185, Italy \\ E-mail:marco.vignati@roma1.infn.it
}

\begin{abstract}
Large-mass bolometers are used in particle physics experiments to search for rare processes. The energy threshold of such detectors plays a critical role in their capability to search for dark matter interactions and rare nuclear decays. We have developed a trigger and a pulse shape algorithm based on the matched filter technique which, when applied to data from test bolometers of the CUORE experiment, lowered the energy threshold from tens of $\mathrm{keV}$ to the few $\mathrm{keV}$ region. The detection efficiency is in excess of $80 \%$, and nearly all nonphysical pulses are rejected.
\end{abstract}

KEYWORDS: Trigger algorithms; Digital signal processing; Bolometers for dark matter research.

\footnotetext{
${ }^{*}$ Corresponding author.
} 


\section{Contents}

1. Introduction 1

2. Experimental setup 2

3. Data filtering

4. Trigger algorithm

5. Detection efficiency 8

6. Pulse shape identification 9

7. Conclusions 10

\section{Introduction}

Bolometers are detectors in which the energy from particle interactions is converted into heat and measured via the resulting rise in temperature. They provide excellent energy resolution, though their response is slow compared to conventional detectors. These features make them a suitable choice for experiments searching for rare processes, such as neutrinoless double beta decay $(0 v \mathrm{DBD})$ and dark matter (DM) interactions.

The CUORE experiment will search for $0 v \mathrm{DBD}$ of ${ }^{130} \mathrm{Te}$ [1] using an array of $988 \mathrm{TeO}_{2}$ bolometers of $750 \mathrm{~g}$ each. Operated at a temperature of about $10 \mathrm{mK}$, these detectors maintain an energy resolution of a few $\mathrm{keV}$ over their energy range, extending from a few $\mathrm{keV}$ up to several $\mathrm{MeV}$. The measured resolution in the region of interest $(2527 \mathrm{keV})$ is about $5 \mathrm{keVFWHM}$; this, together with the low background and the high mass of the experiment, determines the sensitivity to the $0 v \mathrm{DBD}$. CUORE could also search for DM interactions, provided that the energy threshold is sufficiently low. DM candidates such as weak interacting massive particles (WIMPs) and axionlike particles (ALPs) [2] are expected to produce signals at energies below $\sim 30 \mathrm{keV}$, and the interaction rate increases as the energy decreases.

The existing CUORE energy threshold, achieved using a trigger algorithm applied to the raw data samples, is of the order of tens of $\mathrm{keV}$. If the threshold were of few $\mathrm{keV}$, the CUORE experiment could be sensitive to DM interactions and thus play an important role in this growing research area. At low energies the detection capability is limited by the detector noise. Electronics spikes, mechanical vibrations, and temperature fluctuations can produce pulses that, if not properly identified, generate nonphysical background. The lower the energy released in the bolometer, the more difficult it is to discriminate between physical and nonphysical pulses. 
In this paper we present a method to lower significantly the energy threshold, and a pulse shape parameter featuring high rejection power even at low energy. The advantage of this method comes from running the trigger and pulse shape algorithms on data that have been previously processed with the matched filter technique [3]. A lower energy threshold can be obtained because, compared to raw data, matched filtered data feature a higher signal to noise ratio. The algorithms need as input the expected shape of the signal and the noise power spectrum of the bolometer. No manual tuning is needed, because all the parameters can be set at optimal values automatically. Application of this technique to data from a test detector shows that the energy threshold can be lowered to a few $\mathrm{keV}$, and that nonphysical pulses can be efficiently identified. The technique we have developed can be used with any kind of bolometric detector.

\section{Experimental setup}

The algorithms developed in this paper were tested on two bolometers operated by the CUORE collaboration at the LNGS underground laboratory. The main purpose of the test was to check one of the first production batches of CUORE crystals [ [- In the following we will describe our studies taking the first bolometer as example, while the final results will be shown for both.

A CUORE bolometer is composed of two main parts, a $\mathrm{TeO}_{2}$ crystal and a neutron transmutation doped Germanium (NTD-Ge) thermistor [5, 6]. The crystal is cube-shaped $\left(5 \times 5 \times 5 \mathrm{~cm}^{3}\right)$ and held by Teflon supports in copper frames. The frames are connected to the mixing chamber of a dilution refrigerator, which keeps the system at the temperature of $\sim 10 \mathrm{mK}$. The thermistor is glued to the crystal and acts as thermometer. When energy is released in the crystal, its temperature increases and changes the thermistor's resistance [7, 8, 9]. The thermistor is biased with a constant current, and the voltage across it constitutes the signal [10]. Usually, a Joule heater is also glued to the crystal. It is used to inject controlled amounts of energy into the crystal, to emulate signals produced by particles [11, 12].

The typical response of CUORE bolometers to particles impinging on the crystal is of order $100 \mu \mathrm{V} / \mathrm{MeV}$. The signal frequency bandwidth is $0-20 \mathrm{~Hz}$, while the noise components extend to higher frequencies. The signal is amplified, filtered with a 6-pole active Bessel filter with a cut-off frequency of $12 \mathrm{~Hz}$ and $20 \mathrm{~Hz}$ for the first and the second bolometer, respectively, and then acquired with an 18-bit ADC with a sampling frequency of $125 \mathrm{~Hz}$. Given the low event rate and the small frequency bandwidth of the signal, it is possible to save the complete continuous data stream of the detector voltages, so that it can be reprocessed offline. An online software trigger is used as well: when it fires, a fixed number of data samples is saved. There are three kinds of triggers: those caused by particle-like pulses, random waveform samplings, and those generated by the Joule heater. Each event is tagged with a flag that indicates the trigger type. The standard online particle trigger algorithm is based on a simple derivative approach: it fires when the slope of the signal remains above threshold for a certain amount of time. In the measurements discussed in this paper, the energy threshold reached with this algorithm was $11 \mathrm{keV}$ on the first bolometer and $40 \mathrm{keV}$ on the second one.

A typical signal produced by an $88 \mathrm{keV} \gamma$ particle generated by a ${ }^{127 m} \mathrm{Te}$ de-excitation in the crystal is shown in Fig. 11. The length of the acquisition window was 5.008 s, corresponding to $M=626$ samples. The energy calibration was made by exposing the bolometer to a ${ }^{232} \mathrm{Th}$ 


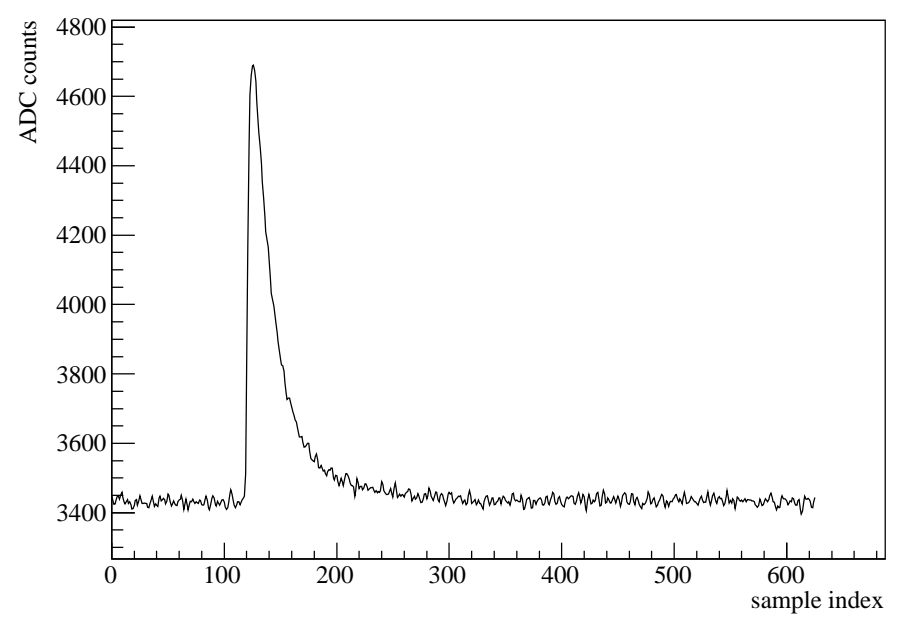

Figure 1. Signal produced by a $88 \mathrm{keV} \gamma$ particle fully absorbed in the detector. The signal is sampled at $125 \mathrm{~Hz}$ and the length of the window corresponds to $5.008 \mathrm{~s}$ (626 samples).

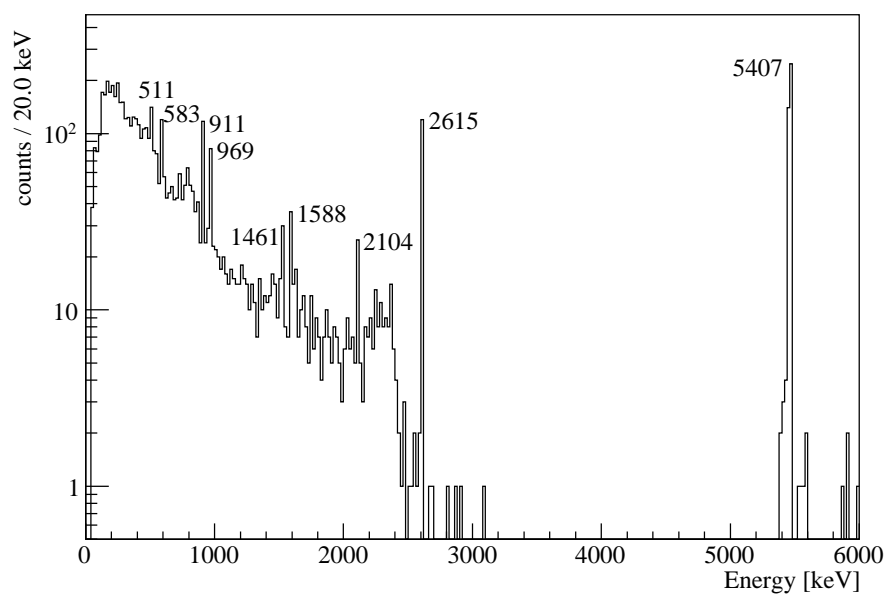

Figure 2. Energy spectrum of the calibration measurement. All lines are generated by the ${ }^{232} \mathrm{Th}$ calibration source except for the lines at $1461 \mathrm{keV}$, due to ${ }^{40} \mathrm{~K}$ contamination of the refrigerator, and at $5407 \mathrm{keV}$, due to ${ }^{210} \mathrm{Po}$ contamination in the $\mathrm{TeO}_{2}$ crystal.

source; for what concerns our studies, the calibration function can be considered linear at energies below $100 \mathrm{keV}$. For the first bolometer the conversion factor from ADC counts to energy is $\sim 15 \mathrm{ADC}$ counts $/ \mathrm{keV}$. The acquired calibration spectrum is shown in Fig. 2. In it, the most prominent peaks of the ${ }^{232} \mathrm{Th}$ source are visible, together with two lines at $1461 \mathrm{keV}$ and $5407 \mathrm{keV}$, which are due to ${ }^{40} \mathrm{~K}$ contamination of the refrigerator and ${ }^{210}$ Po contamination of the crystal bulk, respectively. The ${ }^{210} \mathrm{Po}$ line decays with a half-life of 138 days and is visible in recently-produced crystals. In the data analyzed in this paper, the rate of the ${ }^{210}$ Po line was about $10 \mathrm{mHz}$ and significantly affected the detection efficiencies at low energies, as will be shown in the next sections. 


\section{Data filtering}

The trigger and the pulse shape identification algorithms presented in this paper operate on data samples processed using the matched filter technique [3]. This filter is designed to estimate the amplitude of a signal, maximizing the signal to noise ratio. The transfer function is matched to the signal shape, and pulses with different shape are suppressed. The noise power spectrum of the detector, $N\left(\omega_{k}\right)$, and the signal shape, $s_{i}$, are needed to build the transfer function:

$$
H\left(\omega_{k}\right)=h \frac{s^{*}\left(\omega_{k}\right)}{N\left(\omega_{k}\right)} e^{-\jmath \omega_{k} i_{M}},
$$

where $s\left(\omega_{k}\right)$ is the Discrete Fourier Transform (DFT) of $s_{i}, i_{M}$ is the maximum position of $s_{i}$ in the acquisition window, and $h$ is a normalization constant that leaves unmodified the amplitude of the signal:

$$
h=1 / \sum_{k} \frac{\left|s\left(\omega_{k}\right)\right|^{2}}{N\left(\omega_{k}\right)} .
$$

The noise power spectrum at the filter output can be estimated as

$$
N_{f}\left(\omega_{k}\right)=h^{2} \frac{\left|s\left(\omega_{k}\right)\right|^{2}}{N\left(\omega_{k}\right)},
$$

from which the resolution $\sigma_{f}$ can be derived:

$$
\sigma_{f}^{2}=\sum_{k} N_{f}\left(\omega_{k}\right)=h
$$

The estimation of $N\left(\omega_{k}\right)$ is made by averaging the power spectrum of a large set of data windows not containing signals, while the signal shape $s_{i}$ is obtained by averaging a set of particle signals selected from the calibration measurement in the $1-3 \mathrm{MeV}$ range. The filtered average pulse is symmetric and has the same amplitude as the original (see Fig. 3, left). On the other hand, noise frequencies with low signal to noise ratio are suppressed (see Fig. 3 , right). The resolution before the filter, $\sigma$, is $1.1 \mathrm{keV}$ and, according to Eq. 3.4, is reduced by the filter to $\sigma_{f}=0.3 \mathrm{keV}$.
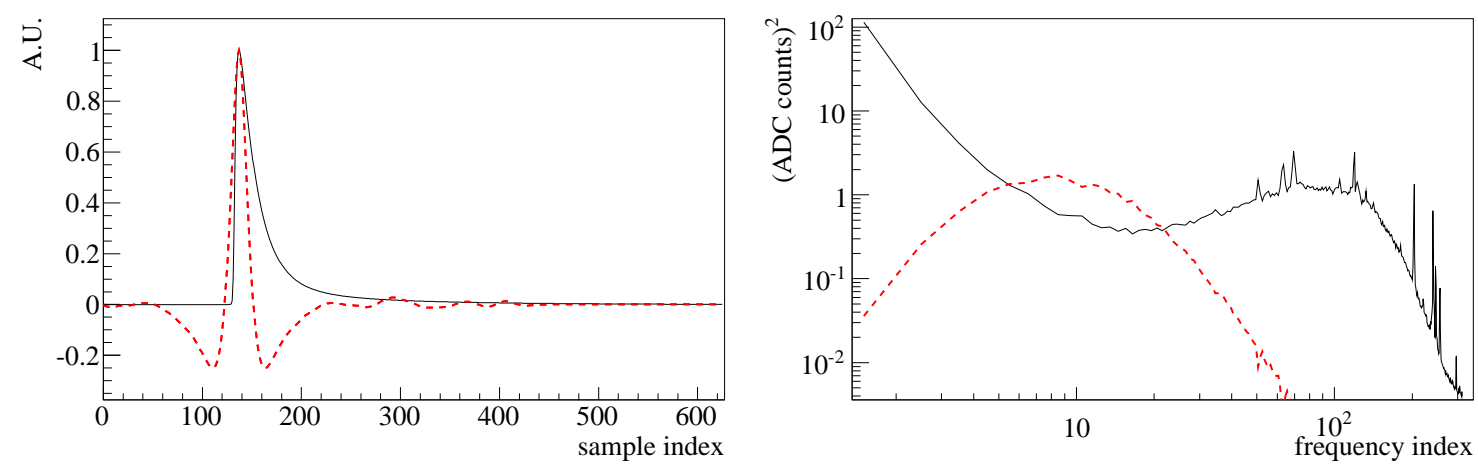

Figure 3. Average pulse (left) and noise power spectrum (right) of the detector, before (solid black line) and after (dashed red line) the filter. The filtered average pulse is symmetric and has the same amplitude as the original. The filtered noise is enhanced in the signal frequency bandwidth and suppressed elsewhere. 
The filtering of the data samples can be implemented in two ways, in the time domain or in the frequency domain. In the time domain one has to implement a finite impulse response (FIR) filter whose kernel is the DFT of Eq. 3.1. In the frequency domain, data samples first have to be transformed using DFT, then multiplied by Eq. 3.1, and then transformed back in the time domain. The number of floating-point operations of the two methods is of the same order of magnitude, namely $O\left(M^{2}\right)$. However, in the frequency domain convolution, FFT algorithms can be used in place of the standard DFT, reducing the number of operations from $O\left(M^{2}\right)$ to $O(M \log M)$. In view of a large scale application, we chose to filter the data in the frequency domain.

DFT algorithms assume that the window to be filtered is periodic. If the input data are not periodic, the convolution will falsely pollute the left side of the window with data from the right side and vice versa. This problem is known as "DFT convolution wraparound" [13] and can be solved by padding the response function of the filter with zeros, such that a slice of the window is correctly filtered. We follow these steps to build the transfer function:

1. Compute the matched filter transfer function $H\left(\omega_{k}\right)$ of length $M$ using the average signal shape $s_{i}$ and the noise power spectrum $N\left(\omega_{k}\right)$, both of length $M$.

2. Transform $H\left(\omega_{k}\right)$ into the time domain, obtaining the response function $H_{i}$ of length $M$.

3. Insert $M$ zeros in the middle of $H_{i}$, obtaining the response function $H_{i}^{d}$ of length $2 M$.

4. Smooth $H_{i}^{d}$ in proximity of the zero insertion, obtaining the smoothed response function $H_{i}^{d s}$.

5. Transform $H_{i}^{d s}$ into the frequency domain, obtaining the final transfer function $H^{d s}\left(\omega_{k}\right)$, of length $2 M$.

The smoothing of $H_{i}^{d}$ is needed to avoid the Gibbs phenomenon [14]: if $H_{i}^{d}$ does not approach zero where the zeros will be inserted, a discontinuity will be created, introducing fake oscillations in its DFT. The smoothing is made by multiplying $H_{i}^{d}$ by a quarter-period cosine shape. The cosine period is $L=120$ samples, and the quarter-period that goes from one to zero is used to smooth

$H_{i}^{d}$ at the left of the zero insertion, while the quarter-period that goes from zero to one is used to smooth $H_{i}^{d}$ at the right of the zero insertion.

When filtering a data sample of length $2 M$, the first and the last $M / 2$ samples will be spoiled while the $M$ middle samples will be correctly filtered (Fig. $\llbracket$ ). The continuous data flow is processed by filtering windows of length $2 M$ overlapped by $M$ and taking the $M$ middle samples of each window as output. The concatenation of the middle samples produces a continuous flow of correctly filtered data (Fig. 5).

\section{Trigger algorithm}

Some slices of the filtered data are shown in Fig. 6 and exhibit the following features:

1. The noise fluctuations are reduced.

2. The baseline is zero. 

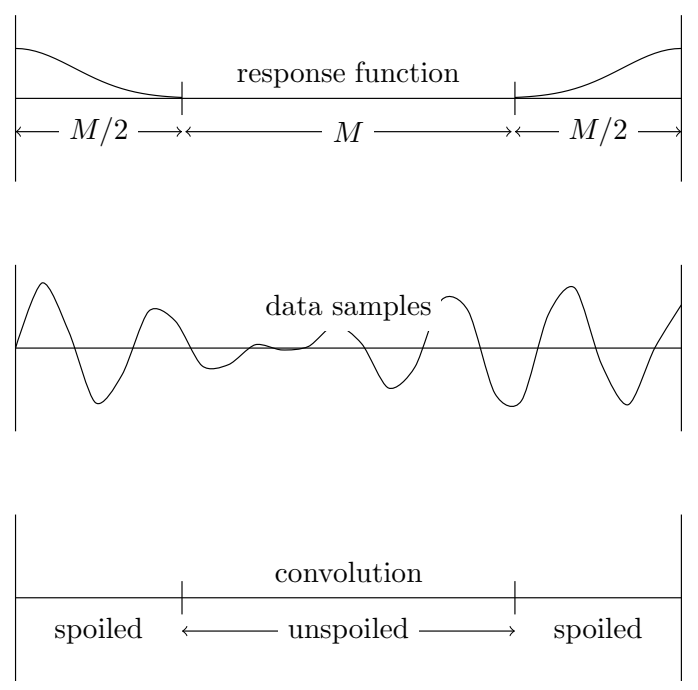

Figure 4. Padding of the filter response function. To avoid the DFT wraparound problem the original response function is padded in the middle with a number of zeros equal to its length. In this way the product of the response function DFT and of the data samples DFT produces a correct convolution in correspondence of the response function zeros.

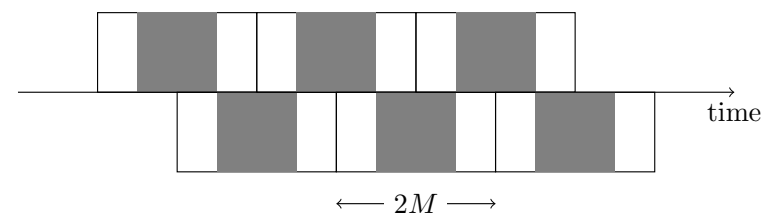

Figure 5. Filtering of the continuous data flow. Each window has size $2 M$ (white rectangle), and the $M$ middle samples are correctly filtered (gray rectangle). The windows are overlapped by $M$, such that the $M$ middle samples of each window are concatenated.

3. The filter is sensitive to the shape of the expected signal and pulses with different shape are suppressed.

Pulses are triggered when the data samples exceed a positive threshold, and a new trigger is possible when the data samples return below threshold, or after that a local maximum is found. The trigger threshold $\theta$ is defined in terms of number of sigma of the filtered noise $\left(\sigma_{f}\right)$, which is known a priori (see Eq. 3.4). The triangles in Fig. 6 show the found triggers and their positions, using the threshold $\theta=4.5 \sigma_{f}$. The trigger position is then shifted with respect to the maximum, so that it lies in the middle of the rise of the pulse. This operation is needed since in the data analysis the trigger is expected to lie before the maximum of a signal. The size of the shift back is fixed and is computed from the time difference between the middle of the rise and the maximum of the non-filtered average pulse.

Although the matched filter dramatically simplifies the implementation of trigger algorithms, there is a drawback. When high-energy pulses are triggered, a set of secondary pulses is seen. This is due to the fact that the filtered pulse has two symmetric lobes above zero, that would be marked as signal by the trigger (see Fig. 7). This problem is solved as follows. The amplitude above zero of the side lobes is a constant fraction $\alpha$ of the amplitude of the main lobe, and is estimated from 

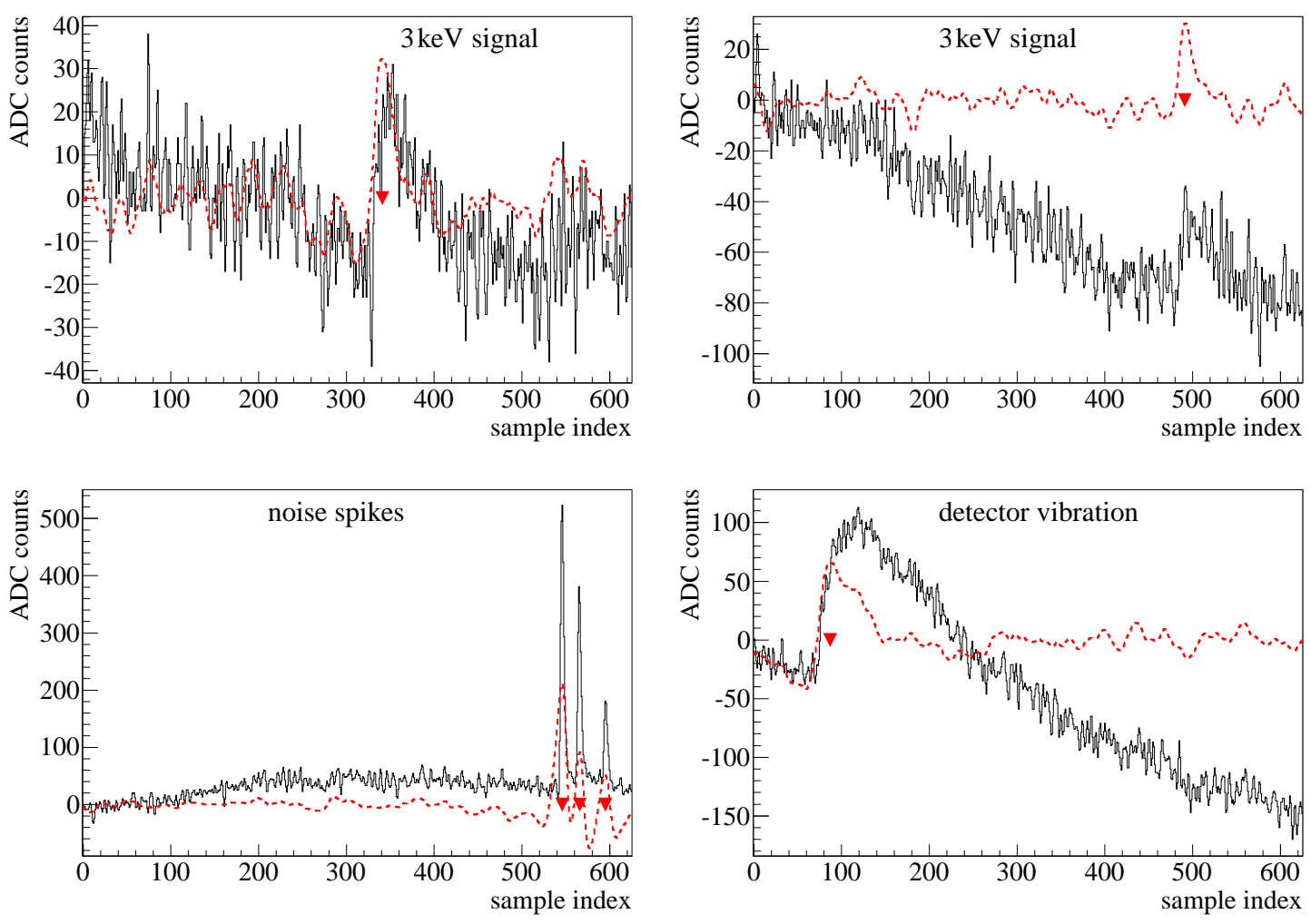

Figure 6. Windows of data samples containing pulses. The raw data (solid black line) have been shifted such that the first sample has zero ADC counts; the filtered data (dashed red line) are not modified. Red triangles identify the pulses detected by the trigger algorithm. The filter removes the baseline drifts and suppresses pulses with different shapes than the expected signal.
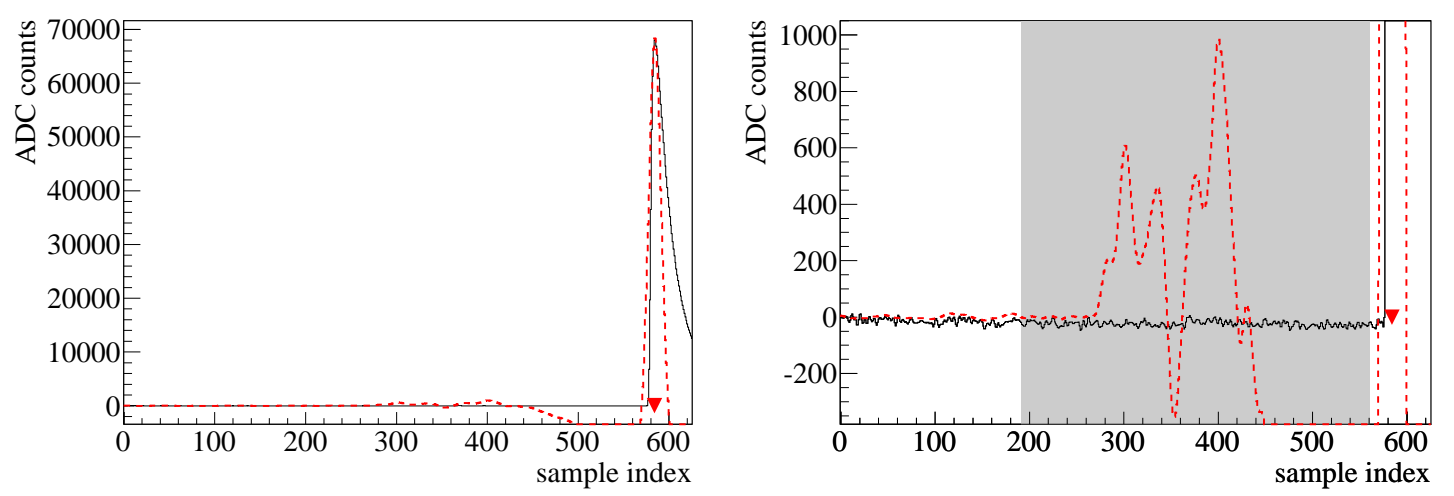

Figure 7. Filtering of a high-energy signal $(5407 \mathrm{keV} \alpha)$. When a high energy pulse occurs (left) the side lobes of the filtered signal exceed the threshold, and fake signals could be triggered. To avoid this, the regions corresponding to the lobes are vetoed and no fake trigger is saved. In the right picture: the left lobe of the pulse, and the vetoed region (gray band).

the filtered average pulse. When a signal with amplitude $A>\theta / \alpha$ is triggered, the data samples corresponding to the expected positions of the side lobes are vetoed, and no trigger is allowed 
in those regions (see Fig. 7). This is an important source of inefficiency for the measurements discussed in this paper, since the ${ }^{210}$ Po contaminations were high and the amount of dead time was dominated by the rate of the $5407 \mathrm{keV}$ line. When crystals are sufficiently aged (one or two years since production), the rate is so low that the dead time is negligible.

\section{Detection efficiency}

To measure the detection efficiency, an energy scan with the external heater was performed using sequences of pulses from the $\mathrm{keV}$ region up to $\sim 100 \mathrm{keV}$ (Fig. \&).

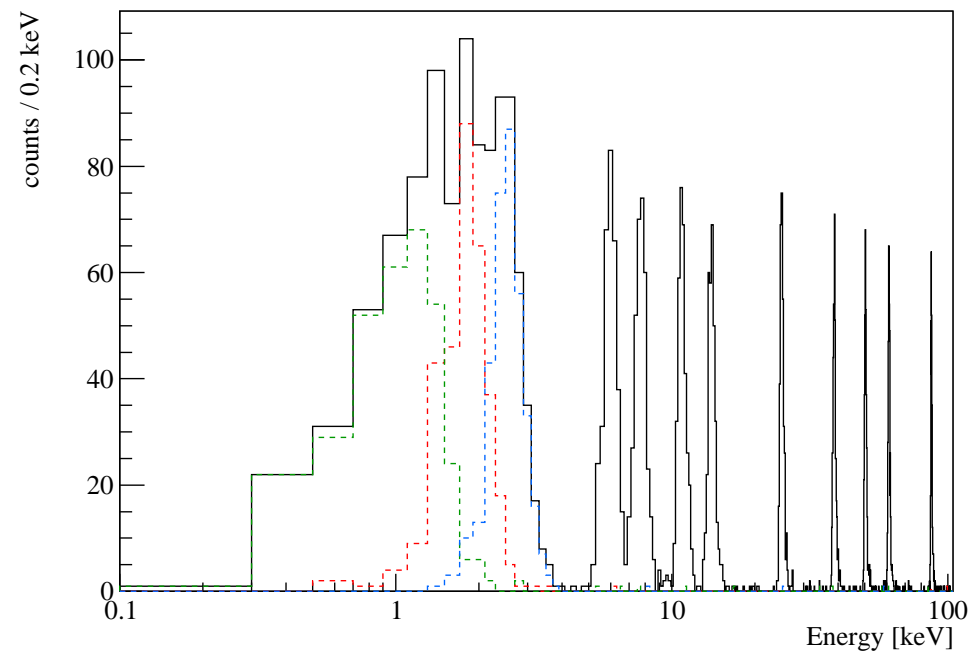

Figure 8. Energy spectrum of heater pulses in the scan measurement. Only pulses flagged as "heater" by the DAQ are shown. Below $\sim 3 \mathrm{keV}$, pulses were fired at 3 different energies.

Comparing the trigger output with the DAQ heater flag, each pulse is considered detected if:

1. The trigger fired on a data sample in a time window that goes from 20 samples before the flag to 30 samples after.

2. The energy obtained from the matched filter amplitude is compatible with the energy expected for the specific heater pulse.

In order to verify this last condition, energy spectra of events related to each heater amplitude set are considered. In each spectrum there is a single peak and some outlier in which the matched filter failed to assign the correct amplitude, due to noise fluctuations or pile-up phenomena. Using the resolution $\sigma_{f}$ in Eq. 3.4, all the events not included in a $2 \mathrm{FWHM}$ energy window $\left(4.7 \sigma_{f}\right)$, centered on the mean energy of the peak, are flagged as not detected. The detection efficiency is then estimated as the ratio of the events within this energy window and the total number of heater pulses fired at that energy.

The measured efficiency, compared with the one of the standard trigger, is shown in Fig. 9 for the two bolometers. The efficiency increases with energy and reaches a plateau. In the first 

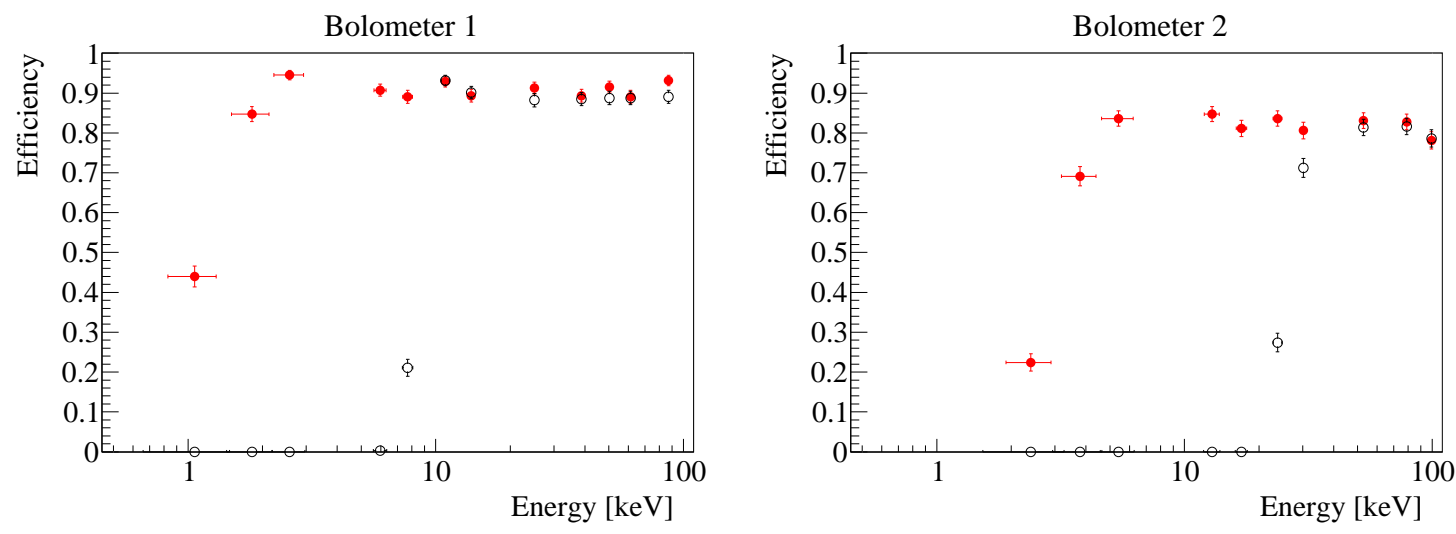

Figure 9. Detection efficiency computed via the heater scan for the standard trigger (open black circles) and the new trigger (solid red circles). The efficiency increases with energy, reaching a plateau. In the first bolometer the plateau is reached at $\sim 11 \mathrm{keV}$ by the standard trigger and at $\sim 2.5 \mathrm{keV}$ by the new trigger. In the second bolometer the plateau is reached at $30 \div 50 \mathrm{keV}$ by the standard trigger and at $\sim 5 \mathrm{keV}$ by the new trigger.

bolometer the plateau efficiency amounts to $90 \%$ and is reached at $\sim 2.5 \mathrm{keV}$. This $10 \%$ efficiency loss is compatible with the veto dead time due to the ${ }^{210} \mathrm{Po}$ line rate. The same efficiency plateau is reached by the standard trigger at $\sim 11 \mathrm{keV}$. In the second bolometer the improvement is even more evident: the $84 \%$ plateau is reached at $30 \div 50 \mathrm{keV}$ by the standard trigger and at $\sim 5 \mathrm{keV}$ by the new trigger.

\section{Pulse shape identification}

As previously stated, the matched filter is sensitive to pulses having the same shape as the expected signal. When a pulse with different shape occurs, its filtered amplitude is suppressed and the filtered shape also is different from the expectation (see Fig. 6). To suppress fake signals we implemented a pulse shape indicator. This indicator is not used to determine the presence of a pulse, but can be used in the data analysis to reduce the nonphysical background.

The algorithm is relatively simple. A triggered pulse is fitted using a cubic spline of the filtered average pulse, and the $\chi^{2} /$ ndf of the fit is used as the shape indicator. The cubic spline is necessary to produce a continuous function and to fit fractional time delays. The fitted parameters are the amplitude and the position of the pulse, while the baseline is fixed at zero. Since the matched filter is itself a fit of the data samples, this fit only serves to remove the digitization effects and to estimate the $\chi^{2}$. Before fitting, the maxima of the fit function and of the triggered pulse are aligned. The pulse position is then varied in the range $[-1,1]$ samples, which is the maximum shift due to the digitization. The amplitude is varied in the range $[A, A(1+\varepsilon)]$, where $A$ is the amplitude of the filtered signal and $\varepsilon$ is the maximum allowed spread in amplitude due to the digitization. This parameter is estimated from the filtered average pulse, computing the difference between the amplitude of the maximum (that is equal to one) and the amplitude of the consecutive sample. Some fits to pulses triggered on the first bolometer are shown in Fig. 10. The fit range $L$ corresponds to 

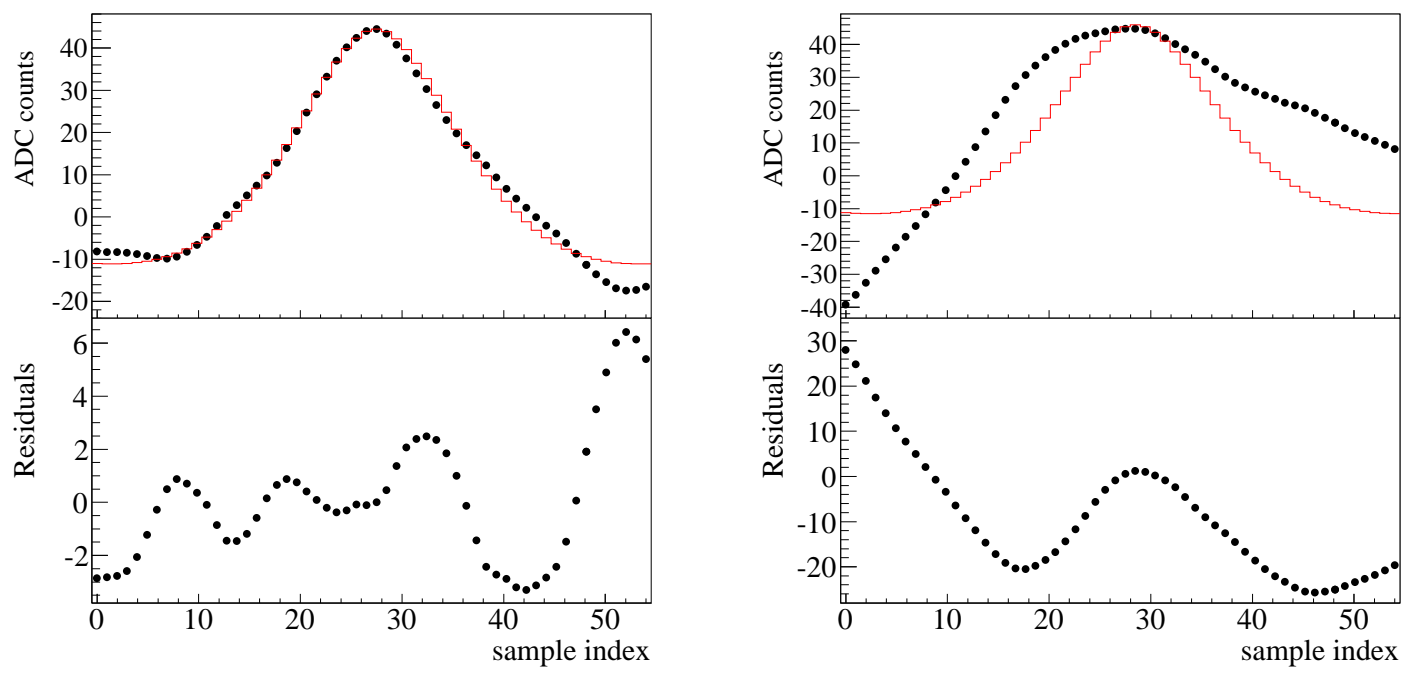

Figure 10. Fits of pulses triggered on the first bolometer; points represent data and residuals, solid lines represent the fit functions. Left: an event in the $3-4 \mathrm{keV}$ region, $S I=0.5$. Right: another event in the $3-4 \mathrm{keV}$ region, $S I=23$.

four FWHM of the filtered average pulse. Finally, the shape indicator (SI) is computed as

$$
\mathrm{SI}=\sum_{i=0}^{L-1} \frac{\left(y_{i}^{f}-f_{i}\right)^{2}}{\sigma_{L}^{2}(L-2)}
$$

where $y^{f}$ is the filtered signal, $f$ is the minimized fit function, and $\sigma_{L}$ is the amount of noise expected in a window of length $L$. The error of each point, in fact, is not the integral error of the entire filtered window in Eq. 3.4, but it is smaller since low frequencies are not seen in a short window:

$$
\sigma_{L}^{2}=\sum_{k=M / L}^{M-M / L} h^{2} \frac{\left|s\left(\omega_{k}\right)\right|^{2}}{N\left(\omega_{k}\right)} .
$$

It must be remarked that, even though Eq. 6.1 has the form of a $\chi^{2}$, it does not follow a true $\chi^{2}$ distribution. Although the expected value is still 1 , the variance is not $2 /(L-2)$ since the noise fluctuations are correlated at the filter output (see residuals in Fig. 10, left).

The distribution of SI versus energy from 4.6 days of data acquired without calibration sources is shown for both bolometers in Figs. 11-12. The signal region is well separated, and nearly all the non-physical pulses can be removed with a cut on this variable. We do not discuss here the optimization of this cut, which depends on a specific analysis.

\section{Conclusions}

We present a method to lower significantly the energy threshold of bolometric detectors, and a pulse shape parameter featuring high rejection power even at low energy. This result is achieved by running the trigger and pulse shape algorithms on data that have been previously processed with the matched filter technique. All the input parameters required by these algorithms can be set at optimal values automatically and no manual tuning is needed. 

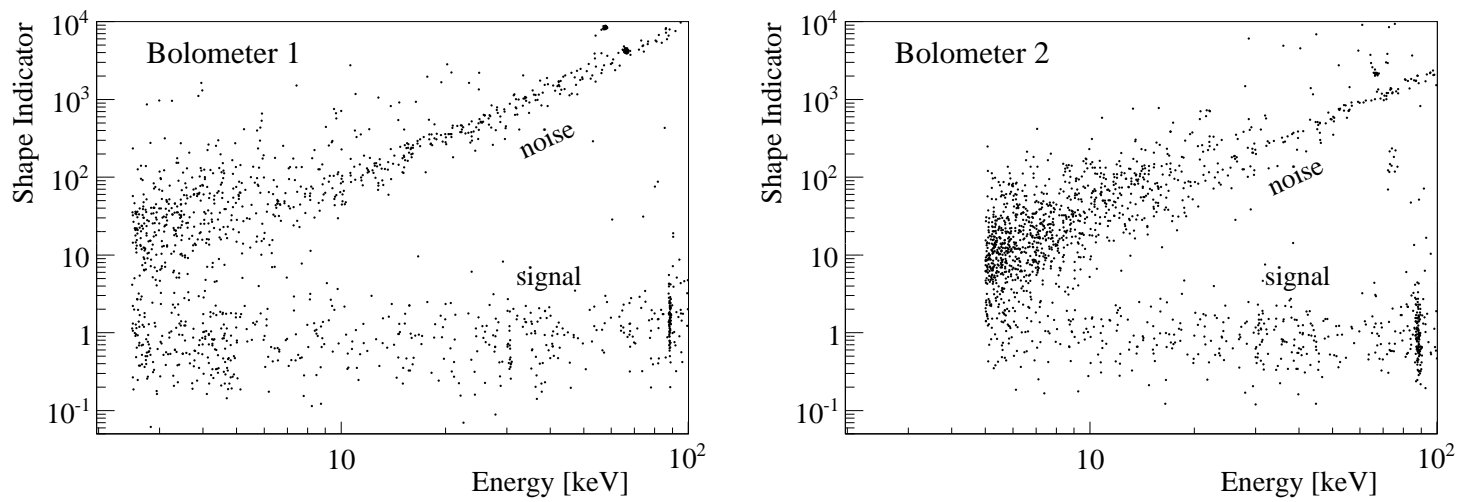

Figure 11. Shape indicator as a function of energy for the first and the second bolometer. The band at low $\mathrm{SI}$ is populated by signal events, where the lines at $30 \mathrm{keV}$ (X-ray from Te) and at $88 \mathrm{keV}$ ( $\gamma$ from ${ }^{127 m} \mathrm{Te}$ ) are visible. At higher values there are triggered mechanical vibrations and spikes.
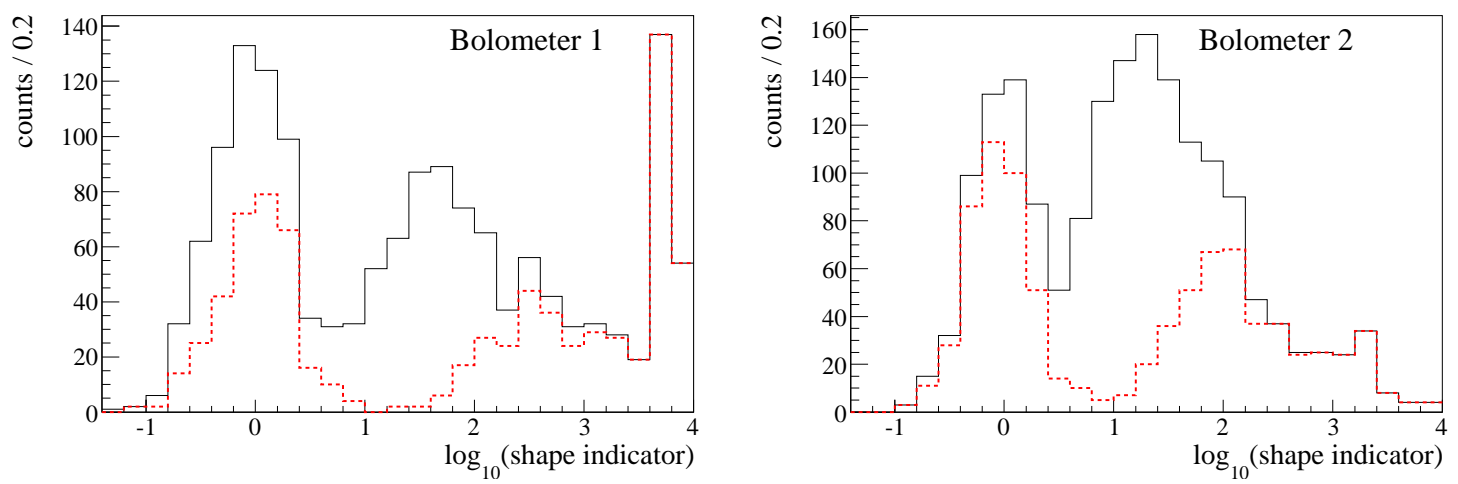

Figure 12. Shape indicator distribution for the first and the second bolometer. The distribution of all the events in the range $2.5-100 \mathrm{keV}$ for the first bolometer, and in the range $5-100 \mathrm{keV}$ for the second bolometer (black solid line), shows a good separation of the signal region (peak around $\log _{10}(\mathrm{SI})=0$ ), which is even more evident if only the events in the range $10-100 \mathrm{keV}$ are selected (red dashed line).

The application to two $\mathrm{TeO}_{2}$ bolometers lowered their thresholds from 11 and $40 \mathrm{keV}$ to 2.5 and $5 \mathrm{keV}$, respectively, with a detection efficiency in excess of $80 \%$. The pulse shape algorithm we developed allows the rejection of nonphysical pulses, such as spikes and temperature fluctuations. With these tools, the $\mathrm{TeO}_{2}$ bolometers of the CUORE experiment will be able to search for new physics at low energies, such as rare nuclear decays and dark matter interactions.

\section{Acknowledgments}

We are very grateful to the members of the CUORE collaboration, in particular to T. Banks, C. Brofferio, Y.G. Kolomensky and M. Pavan for their valuable suggestions, and to F. Ferroni and M. Pallavicini for supporting this work. We wish to thank S. Frasca for fruitful discussions. 


\section{References}

[1] R. Ardito et. al., CUORE: A cryogenic underground observatory for rare events, arXiv:hep-ex/0501010 (2005) [hep-ex/0501010].

[2] G. Bertone, D. Hooper, and J. Silk, Particle dark matter: evidence, candidates and constraints, Phys. Rept. 405 (2005) 279, hep-ph/0404175.

[3] E. Gatti and P. F. Manfredi, Processing the signals from solid state detectors in elementary particle physics, Riv. Nuovo Cim. 9N1 (1986) 1.

[4] C. Arnaboldi et. al., Production of high purity $\mathrm{TeO}_{2}$ single crystals for the study of neutrinoless double beta decay, J. Cryst. Growth 312 (2010), no. 202999.

[5] N. Wang, F. C. Wellstood, B. Sadoulet, E. E. Haller, and J. Beeman, Electrical and thermal properties of neutron-transmutation-doped Ge at 20 mK, Phys. Rev. B 41 (1990), no. 63761.

[6] K. M. Itoh et. al., Neutron transmutation doping of isotopically engineered Ge, Appl. Phys. Lett. 64 (1994) 2121.

[7] N. F. Mott, Localized states in a pseudogap and near extremities of conduction and valence bands, Phil. Mag. 19 (1969) 835.

[8] A. Efros and B. Shklovskii, Electronic properties of doped semiconductors, p. 202. Springer-Verlag, Berlin, 1984.

[9] K. M. Itoh et. al., Hopping conduction and metal-insulator transition in isotopically enriched neutron-transmutation-doped ${ }^{70}$ Ge:Ga, Phys. Rev. Lett. 77 (1996), no. 194058.

[10] C. Arnaboldi et. al., The programmable front-end system for CUORICINO, an array of large-mass bolometers, IEEE Trans. Nucl. Sci. 49 (2002) 2440.

[11] A. Alessandrello et. al., Methods for response stabilization in bolometers for rare decays, Nucl. Instr. Meth. in Phys. Res. A. (1998), no. 412454.

[12] C. Arnaboldi, G. Pessina, and E. Previtali, A programmable calibrating pulse generator with multioutputs and very high stability, IEEE Trans. Nucl. Sci. 50 (2003) 979.

[13] W. H. Press, S. A. Teukolsky, W. T. Vetterling, and B. P. Flannery, Numerical recipes in C (2nd ed.): the art of scientific computing. Cambridge University Press, New York, NY, USA, 1992.

[14] J. Gibbs, Fourier's series, Nature 59 (1898) 200. 